\title{
Nonadiabatic processes involving quantum-like and classical-like coordinates with applications to nonadiabatic electron transfers
}

\author{
R. A. Marcus \\ Noyes Laboratory of Chemical Physics, ${ }^{\text {a) }}$ California Institute of Technology, Pasadena, California 91125
}

(Received 4 June 1984; accepted 5 July 1984)

\begin{abstract}
Nonadiabatic processes may involve both classical-like and quantum-like coordinates. A semiclassical analysis is used to treat the contribution of the former to the Franck-Condon factor in the reaction rate expression, thereby avoiding the usual harmonic oscillator approximation. Microcanonical and canonical rate constants are calculated, yielding an expression which includes contributions from both types of coordinates. The results are applied to nonadiabatic electron transfer reactions in solution, and show how $\Delta G^{\circ}$ enters the final rate expression, even though $\Delta E^{0}$ is present in the initial Golden Rule nonadiabatic formula. This result avoids an approximation which has arisen in the nonadiabatic electron transfer literature.
\end{abstract}

\section{INTRODUCTION}

In nonadiabatic processes, some of the degrees of freedom may be treated as largely classical, and for them it is useful to simplify the usual Golden Rule expression for the transition rate of the process. One such example occurs in nonadiabatic electron transfer reactions in solution. In electron transfer reactions the many orientational coordinates of the solvent molecules are typically classical-like and play an important role in electron transfer reactions, ${ }^{1-3}$ considerably influencing their rate.

One purpose of the present paper is to provide insight and justification for a physically reasonable but somewhat ad hoc procedure which has sometimes been used for nonadiabatic electron transfers. ${ }^{4-6}$ In that procedure the quantum Franck-Condon overlap factors for some coordinates are combined with a classical treatment ${ }^{2,3}$ for the rearrangement of the remaining coordinates, typically the orientational coordinates of the solvent molecules.

In the final theoretical expression for the reaction rate, sometimes $\Delta G^{0}$ but more frequently (e.g., Refs.7-11) $\Delta E^{0}$ is written, where $\Delta G^{0}$ is the standard free energy of reaction. $\Delta E^{0}$ is the standard energy change of reaction, which occurs in the Golden Rule expression for nonadiabatic processes. The appearance of $\Delta E^{\circ}$, instead of $\Delta G^{\circ}$, in the relevant final equations will result from using in such expressions a harmonic oscillator approximation for all the classical-like coordinates. For harmonic oscillators having the same frequencies for reactants as for products, $\Delta S^{\circ}$ vanishes and so $\Delta E^{0}$ and $\Delta G^{0}$ become equal. ${ }^{12}$ For reactions in solution, however, the harmonic oscillator model is inadequate for treating the orientations of the solvent molecules. There can be very large entropic changes accompanying reaction, for example. This approximation is avoided in the present paper by the use of semiclassical theory and generalized (curvilinear) coordinates.

In simplifying the nonadiabatic expression we retain that part of the Franck-Condon factor which relates to any highly quantized degrees of freedom and only use semiclassical theory to convert the remaining part to a classical version. The final result also provides a simple extension of an expression used for "surface hopping" ${ }^{33}$ between two elec-

\footnotetext{
a) Contribution No. 7039 .
}

tronic surfaces via classical trajectories, by allowing some coordinates to be treated purely quantum-like.

\section{THEORY}

The Golden Rule expression for the unimolecular rate constant $k_{\alpha \beta}$ for a nonadiabatic transition $\alpha i \rightarrow \beta f$ from electronic state $\alpha$ to electronic state $\beta$ and from a quantum state $i$ of the nuclear motion on surface $\alpha$ to a state $f$ on surface $\beta$ is

$$
k_{\alpha \beta}=\frac{2 \pi V^{2}}{\hbar}\left|\left\langle\varphi_{f} \mid \varphi_{i}\right\rangle\right|^{2} \delta\left(E_{f}-E_{i}+\Delta E^{0}\right),
$$

where $V$ is the electronic matrix element for the transition (the Condon approximation is made), $f$ denotes the set of quantum numbers $\left(f_{1} \cdots f_{M}\right)$ for the nuclear motion in electronic state $\beta$ ( $M$ coordinates), $i$ denotes those $\left(i_{1} \cdots i_{M}\right)$ for that motion in electronic state $\alpha, E_{f}$ and $E_{i}$ are the corresponding energies, in excess of the zero-point energy of the $\beta$ state and of the $\alpha$ state, respectively, and $\Delta E^{0}$ is the standard energy of the reaction for process $\alpha \rightarrow \beta$ at $0 \mathrm{~K}$. Equation (1) has been extensively used in the nonadiabatic electron transfer literature, e.g., Refs. $4-10$. In that case, $\alpha$ and $\beta$ denote electronic states of the reactants and of the products, respectively.

We shall suppose that of the $M$ degrees of freedom the first $N$ are classical-like and write

$$
\varphi_{i}=\psi_{i} \chi_{I}, \quad \varphi_{f}=\psi_{f} \chi_{F},
$$

where $\varphi_{i}$ refers to $i=1, \ldots, N, \chi_{i}$ to $I=i=N+1, \ldots, M$, and $\psi_{f}$ and $\chi_{F}$ to $f=1, \ldots, N$ and $F=f=N+1, \ldots, M$, respectively.

It is convenient to denote that part of the energies $E_{i}$ and $E_{f}$ in Eq. (1) associated with the $N$ classical-like coordinates by $\epsilon_{i}$ and $\epsilon_{f}$ and the part associated with the remaining coordinates by $E_{I}$ and $E_{F}$, respectively. We define $\Delta E_{I F}^{0}$ as the effective $\Delta E^{0}$ for a transition $\alpha I \rightarrow \beta F$ :

$$
\Delta E_{I F}^{0}=\Delta E^{0}+E_{F}-E_{I} .
$$

Equation (1) now becomes

$$
k_{\alpha \beta}=\frac{2 \pi V^{2}}{\hbar}|\langle F \mid I\rangle|^{2}|\langle f \mid i\rangle|^{2} \delta\left(\epsilon_{f}-\epsilon_{i}+\Delta E_{I F}^{0}\right) .
$$

If all the modes of motion were rotational, rather than some being oscillatory, the "primitive" semiclassical wave function $\psi_{i}$ would be a single term. When some or all or the 
modes of motion are oscillatory the phase of the semiclassical wave function has more than one branch, each branch corresponding to a particular set of signs for the $N$ momenta $\left(p_{1} \cdots p_{N}\right)$. The normalized wave function $\psi_{i}$ can then be written as the sum in Eq. (5), using a Van Vleck determinant $t^{14,15}$ for each term:

$$
\psi_{i}=\sum^{a^{-1 / 2}}\left|\frac{\partial^{2} S_{i}}{\partial q \partial i}\right|^{1 / 2} \frac{e^{i S_{i} / \hbar}}{h^{N / 2}},
$$

where the sum is over the various branches of $S_{i}$ and would contain $2^{N}$ terms if all modes of motion were oscillatory, $S_{i}$ is the phase integral $\Sigma_{k} \int p_{k} d q_{k}$, taken from some turning point where all the $p_{k}$ 's vanish, plus various multiples of $\pi /$ 4 , depending on the particular oscillatory branch, e.g., Refs. $14 \mathrm{~b}, 14 \mathrm{~d}, 14 \mathrm{f}$, and $a$ is a determinant of coefficients in the elementary distance expression (Appendix A). $\partial^{2} S_{i} / \partial q \partial i$ denotes an $N \times N$ determinant having elements $\partial^{2} S_{i} / \partial q_{j} \partial i_{k}$ :

$$
\left|\partial^{2} S_{i} / \partial q \partial i\right| \equiv \operatorname{det} \partial^{2} S_{i} / \partial q_{k} \partial i_{j} .
$$

We use this shorthand notation for determinants throughout, as well as an anologous one for differentials and for sets of variables:

$$
d u \equiv d u_{1} \cdots d u_{N}, \quad u \equiv\left(u_{1} \cdots u_{N}\right) .
$$

The normalization factor in Eq. (5) can be verified by first noting that in the product $\psi_{i}^{*} \psi_{i}$ any highly oscillatory terms can be neglected. Only terms in which $S_{i}$ and $S_{i}$ are of the same branch then remain. We obtain

$$
\begin{aligned}
\left\langle i^{\prime} \mid i\right\rangle & =\int \cdots \int \psi_{i}^{*} \psi_{i^{\prime}} \sqrt{a} d q \\
& =\sum \int \exp \left[\sum_{k}\left(\partial S_{i} / \partial i_{k}\right)\left(i_{k^{\prime}}-i_{k}\right) / \hbar\right] d\left(\partial S_{i} / \partial i\right) / h^{N},
\end{aligned}
$$

where the sum $\Sigma$ is again over the branches of $S_{i}$ and where we have used the shorthand notation (7). In Eq. (8) $S_{i}$, in the exponent was expanded about $S_{i}$ and $\left|\partial^{2} S_{i} / \partial q \partial i\right| d q$ was replaced by its equivalent $d\left(\partial S_{i} / \partial i\right)$. Each $\partial S_{i} / \partial\left(i_{k} h\right)$ is the angle variable conjugate to the classical action $i_{k} h^{16}$ and its individual domain is the unit interval. However, different branches in the sum correspond, for oscillatory modes of motion, to different parts of this unit domain. (For any rotational motion the single branch covers the whole unit interval.) Upon removing the sum sign in (8) before the integral we recognize the fact that each angle variable ranges, thereby, over its full $(0,1)$ domain. The right-hand side of Eq. (8) then becomes, on integration, a product of Kronecker deltas $\delta_{i_{1} i_{1}}, \cdots \delta_{i_{k_{k}} i_{k}}$. Thus, the semiclassical $\psi_{i}$ given by Eq. (5) is properly normalized.

We consider next the Franck-Condon factor ${ }^{17}\langle i \mid f\rangle$ using Eq. (5) for $\psi_{i}$ and an analogous expression for $\psi_{f}$. The integral over the coordinates $q$ becomes

$$
\langle i \mid f\rangle=\Sigma \int \ldots \int\left|\frac{\partial^{2} S_{i}}{\partial q \partial i}\right|^{1 / 2}\left|\frac{\partial^{2} S_{f}}{\partial q \partial f}\right|^{1 / 2} e^{i \mid S_{,}-S_{i} / \hbar \hbar} \frac{d q}{h^{N}},
$$

and is evaluated, as is customary for semiclassical FranckCondon factors, by the stationary phase method. The only terms retained in the sum are those where $S_{f}$ and $S_{i}$ belong to the same branch, the remaining terms being highly oscillatory. The stationary phase point is determined by

$$
\frac{\partial}{\partial q_{k}}\left(S_{f}-S_{i}\right)=0
$$

i.e.,

$$
p_{k}^{f}=p_{k}^{i}(k=1, \ldots, N)
$$

and we have

$$
\begin{aligned}
S_{f}-S_{i} \cong & S_{f}^{0}-S_{i}^{0}+\frac{1}{2} \sum_{k_{j}, j} \frac{\partial^{2}}{\partial q_{k} \partial q_{j}} \\
& \times\left(S_{f}-S_{i}\right)\left(q_{k}-q_{k}^{0}\right)\left(q_{j}-q_{j}^{0}\right),
\end{aligned}
$$

where the various quantities on the right are evaluated at the stationary phase point $q=q^{0}$. Integration in Eq. (9) then yields

$$
\begin{aligned}
\langle i \mid f\rangle= & \sum\left[\left|\frac{\partial^{2} S_{i}}{\partial q \partial i}\right|\left|\frac{\partial^{2} S_{f}}{\partial q \partial f}\right| /\left|\frac{\partial^{2}\left(S_{f}-S_{i}\right)}{\partial q^{2}}\right|\right]^{1 / 2} \\
& \times e^{i\left(S_{f}^{0}-S_{i}^{0}\right) / \hbar} / h^{N / 2} .
\end{aligned}
$$

The stationary phase points (10) all occur at the same $q$, but with different combinations of signs of the $p_{k}$ 's. If this $q^{0}$ is the only stationary phase point, the number of terms in the sum is the same as that in Eq. (5), one per branch of $S_{i}$. There may also be stationary phase points at other values of $q$, and if so they are then included in the sum.

Equation (12) can be simplified in a straightforward way to yield ${ }^{18}$

$$
\langle i \mid f\rangle=\sum\left|\frac{\partial p^{i}}{\partial i}\right|^{1 / 2}\left|\frac{\partial q}{\partial f}\right|^{1 / 2} \frac{e^{i W_{f} / \hbar}}{h^{N / 2}},
$$

where $W_{f}$ is the value of $S_{f}-S_{i}$ at the stationary phase point (10) and the sum is again over the branches of $S_{i}$.

From Eqs. (4) and (13) one obtains

$k_{\alpha \beta}=\frac{2 \pi V^{2}}{\hbar h^{N}}|\langle F \mid I\rangle|^{2} \sum\left|\frac{\partial p^{i}}{\partial i}\right|\left|\frac{\partial q}{\partial f}\right| \delta\left(\epsilon_{f}-\epsilon_{i}+\Delta E_{I F}^{0}\right)$.

The rate constant for a process $\alpha I \rightarrow \beta F$, when the initial state of motion of the classical-like coordinates is described by $\left(i_{1} \cdots i_{N}\right)$, is found by integrating Eq. (14) over the $f$ 's. In doing this we first introduce a coordinate system to simplify the resulting expression: The intersection of the two potential energy surfaces $U_{\alpha}(q)$ and $U_{\beta}(q)$ occurs where $U_{\alpha}(q)=U_{\beta}(q)+\Delta E_{I F}^{0}$ and defines an $N-1$ dimensional hypersurface in the $N$-dimensional coordinate space. We define a coordinate $q_{N}$ which is constant on this hypersurface, and which takes on different values for other values of $U_{\beta}(q)-U_{\alpha}(q)$.

Equation (14) is next integrated over the $\left(f_{1}, \ldots, f_{N}\right)$ variables, noting that $|\partial q / \partial f| d f$ equals $d q$. Further, each stationary phase point (10) occurs on the hypersurface just cited, and at that point $\epsilon_{f}-\epsilon_{i}$ equals $U_{\beta}-U_{\alpha}$, since $p_{k}^{i}=p_{k}^{f}$ there for all $p_{k}$ 's. Thus, the $\delta\left(\epsilon_{f}-\epsilon_{i}-\Delta E_{I F}^{0}\right)$ in Eq. (14) can be replaced by $\delta\left(U_{\beta}-U_{\alpha}-\Delta E_{I F}^{0}\right)$. One can then write $d q_{N}$ as $d\left(U_{\beta}-U_{\alpha}\right) /\left[\partial\left(U_{\beta}-U_{\alpha}\right) / \partial q_{N}\right]$ and define the absolute value $S$ of this difference of slopes:

$$
S=\left|\partial\left(U_{\beta}-U_{\alpha}\right) / \partial q_{N}\right| .
$$

In integrating over the $f$ 's in Eq. (14) one notes that $|\partial q / \partial f| d f$ equals $d q$, then uses the $\delta\left(U_{\beta}-U_{\alpha}-\Delta E_{I F}^{0}\right)$ in the integration over $d\left(U_{\beta}-U_{\alpha}\right)$, and so obtains the rate constant for a state $\left(i_{1} \cdots i_{N}\right)::^{2,19}$ 


$$
\begin{aligned}
k\left(i_{1} \cdots i_{N}\right)= & \frac{2 \pi V^{2}}{\hbar}|\langle F \mid I\rangle|^{2} \\
& \times \sum \int \cdots \int \frac{\partial p^{i}}{\partial i} S^{-1} d q_{1} \cdots d q_{N-1} / h^{N} .
\end{aligned}
$$

The microcanonical rate constant $k_{I F}(\epsilon)$ for a given transition $\alpha I \rightarrow \beta F$ is obtained by integrating $k\left(i_{1} \cdots i_{N}\right)$ over all $i_{k}$ 's such that $\epsilon_{i}$ lies in a narrow interval $(\epsilon, \epsilon+d \epsilon)$ and then dividing by the number $f \cdots f d i_{1} \cdots d i_{N}$ of states in this interval, i.e., by $\rho_{\alpha} d \epsilon$, where $\rho_{\alpha}$ is the density of states of the classical-like degrees of freedom in electronic state $\alpha$. One next notes that $\left|\partial p^{i} / \partial i\right| d i$ equals $d p^{i}$, and that when the space of numbers $\left(i_{1} \cdots i_{N}\right)$ is mapped onto the space of $p_{k}^{i}$ 's the various signs of $p_{k}^{i}$ 's, which created the various branches in the sum in Eq. (16), serve to map the $i_{k}$ 's onto all of the relevant $p^{i}$ space, and so the sum in Eq. (16) disappears. We then have

$k_{I F}(\epsilon)=\frac{2 \pi V^{2}|\langle F \mid I\rangle|^{2}}{\hbar \varphi_{\alpha}(\epsilon) d \epsilon \hbar^{N}} \int \ldots \int S^{-1} d p_{1} \cdots d p_{N} d q_{1} \cdots d q_{N-1}$.

The integration in Eq. (17) is restricted to the domain where $\left(q_{1} \cdots q_{N-1}, p_{1} \cdots p_{N}\right)$ lies within the energy shell $(\epsilon, \epsilon+d \epsilon)$, and so the integral is proportional to $d \epsilon$.

To obtain the rate constant at a given temperature $k_{I F}(T)$ for the $\alpha I \rightarrow \beta F$ transition, one multiplies Eq. (17) by $\rho_{\alpha}(\epsilon) \exp (-\epsilon / k T) d \epsilon / Q, Q$ being the partition function for the $N$ classical-like coordinates of the reactant, and extends the integration to all values of the $2 N-1$ variables:

$$
\begin{aligned}
k_{I F}(T)= & \frac{2 \pi V^{2}}{\hbar Q}|\langle F \mid I\rangle|^{2} \int \ldots \int \\
& \times e^{-\epsilon / k T} S^{-1} d p_{1} \cdots d p_{N} d q_{1} \cdots d q_{N-1} .
\end{aligned}
$$

In passing we note that the stationary phase point for a transition $\left(i_{1} \cdots i_{N}\right) \rightarrow\left(f_{1} \cdots f_{N}\right)$ determines a point in the $2 N$-dimensional phase space. When the additional condition on the energy $\epsilon_{f}$ is imposed, via the delta function in Eq. (14), the $i \rightarrow f$ transition corresponds to a point in $2 N-1$ dimensional phase space, a point in the domain in Eq. (17) and (18). The integration over this space corresponds to the integration over all $i$ 's and $f$ 's, subject to the one constraint imposed by a delta function.

Equation (18) is first integrated over the momenta. The kinetic energy in $\epsilon$ is written as $\frac{1}{2} \Sigma_{i, j} g^{i j} p_{i} p_{j}$ and one obtains (Appendix A)

$$
\begin{aligned}
& k_{I F}(T)=\frac{2 \pi V^{2}}{\hbar}|\langle F \mid I\rangle|^{2} \\
& \times \frac{\int \cdots \int e^{-U_{\alpha} k T} S^{-1} \sqrt{a} d q_{1} \cdots d q_{N-1}}{\int \cdots \int e^{-U_{\alpha} / k T} \sqrt{a} d q_{1} \cdots d q_{N}} .
\end{aligned}
$$

The overall rate constant $k(T)$ is obtained by multiplying Eq. (19) by the probability of finding the system in the state $I$ and summing over all $I$ 's and all $F$ 's:

$$
k(T)=\sum_{I, F} k_{I F}(T) e^{-E_{1} / k T} / Q_{I},
$$

where $Q_{I}$ is the partition function $\Sigma_{I} \exp \left(-E_{I} / k T\right)$ of the degrees of freedom associated with the quantum numbers $I$.

The $k(T)$ for the reverse reaction is obtained by interchanging $(I, \alpha)$ with $(F, \beta)$. One can verify that microscopic reversibility is obeyed by these two rate constants, upon noting that $U_{\alpha}$ equals $U_{\beta}+\Delta E_{I F}^{0}$. The ratio of the two rate constants is found to equal, as it should, $\exp \left(-\Delta E^{0} / k T\right)$ times the ratio of the appropriate partition functions.

In the case of an adiabatic reaction one obtains Eq. $(19),{ }^{20}$ but with several differences [cf. Eqs. (33) and (34) of Ref. 20]: the $S^{-1}$ is absent, the $N-1$ dimensional surface element differs slightly from that element in Eq. (19), a consequence of the absence of a $q_{N}$ term in Eq. (17), and a different factor precedes the integral.

To relate Eqs. (19) and (20) to an equation which has been used ${ }^{4-6}$ for treating nonadiabatic electron transfers when some of the degrees of freedom are treated quantum mechanically and some classically, two further steps will be used. We need to relate the integrals in Eq. (19) to a free energy of formation of a nonequilibrium polarization state $\mathrm{s}^{2,3}$ appropriate to the transition state of the reaction, and require, thereby, the two integrals to have the same number of coordinates. This nonequilibrium state is one which is "centered" $" 3,21$ on the intersection hypersurface.

To relate these integrals to this free energy of formation, we first replace the $S^{-1}$ by some average $\left\langle S^{-1}\right\rangle$ which we discuss later, and thus obtain an integral involving $\exp \left(-U_{\alpha} / k T\right) \sqrt{a} d q_{1} \cdots d q_{N-1}$. We next replace this surface distribution by an "equivalent equilibrium distribution", 3,21 by multiplying by $\exp \left(-U_{N} / k T\right) d q_{N}$, integrating over $q_{N}$, and then dividing by a one-dimensional configuration partition function $Q_{N}$ for this motion of $q_{N} \cdot{ }^{22}$ Here, $U_{N}$ is an effective potential energy function which vanishes on the intersection surface $U_{\alpha}=U_{\beta}+\Delta E_{I F}^{0}$, and which serves to convert the surface distribution to a volume distribution centered on the intersection surface, the "e.e.d." of Refs. 3 and 21. We now have

$$
\begin{aligned}
k_{I F}(T)= & \frac{2 \pi V^{2}}{\hbar}|\langle F \mid I\rangle|^{2} \frac{\left\langle S^{-1}\right\rangle}{Q_{N}} \\
& \times \frac{\int \cdots \int e^{-U_{a}^{*} / k T} \sqrt{a} d q_{1} \cdots d q_{N}}{\int \ldots \int e^{-U_{\alpha} / k T} \sqrt{a} d q_{1} \cdots d q_{N}},
\end{aligned}
$$

where

$U_{\alpha}^{*}=U_{\alpha}+U_{N}$.

The possibility of having a large entropy of activation for the reaction is allowed in Eqs. (19) or (21): In the transition state region, i.e., at the intersection of the $U_{\alpha}$ and $U_{\beta}+\Delta E_{I F}^{0}$ potential energy surfaces, the behavior of $U_{\alpha}$ can be very different from its behavior in the region appropriate to the reactants [denominators of Eqs. (19) or (21)]. For example, in an electron transfer reaction the ionic charges of the reactants differ from those of the products, resulting frequently in enormous changes in entropy of solvation. These changes are reflected in a difference in the behavior of $U_{\alpha}$ in the "reactants' region" of the $N$-dimensional coordinate space compared with the behavior of $U_{\beta}$ in the "products' region". This difference is mirrored in the 
behavior of $U_{\alpha}$ on the intersection hypersurface.

An expression derived for the ratio of integrals in Eq. (21), using a "dielectrically unsaturated" approximation for the response of the solvent outside the coordination shells of the reactants to changes in ionic charges, yielded ${ }^{23}$

$$
\frac{\int \cdots \int e^{-U_{a}^{*} / k T} \sqrt{a} d q_{1} \cdots d q_{N}}{\int \ldots \int e^{-U_{\alpha} / k T} \sqrt{a} d q_{1} \cdots d q_{N}}=\exp \left[\frac{-\lambda_{0}}{4 k T}\left(1+\frac{\Delta G_{I F}^{0}}{\lambda_{0}}\right)^{2}\right],
$$

where $\lambda_{0}$ is a quantity in terms of the molecular properties. For example, if dielectric continuum theory is used it depends on the change in charges of the reactants, the ionic radii of separation distance between reactants, and the dielectric properties of the surrounding medium. ${ }^{24}$ If any vibrations are included, in addition, in the classical-like coordinates $\left(q_{1} \cdots q_{N}\right), \lambda_{0}$ includes a contribution from them also and then depends also on the changes in the corresponding equilibrium bond lengths and on the vibration frequencies. ${ }^{25}$ $\Delta G_{I F}^{0}$ in Eq. (23) is $\Delta G^{0}+E_{F}-E_{I}$. That is, it is the effective $\Delta G^{\circ}$ when the system goes from state $I$ of the quantum degrees of freedom of reactants to state $F$ for those of the products.

We turn next to the remaining factor in Eq. $(21)\left\langle S^{-1}\right\rangle /$ $Q_{N}$. We consider first a simple case, namely where $U_{\alpha}$ for each value of $\left(q_{1} \cdots q_{N-1}\right)$ has a term $\frac{1}{2} k^{\prime}\left(q_{N}-a\right)^{2}$ and where $U_{\beta}$ behaves as a displaced oscillator at that $\left(q_{1} \cdots q_{N_{-1}}\right)$, and so has $\frac{1}{2} k^{\prime}\left(q_{N}-b\right)^{2}-\Delta E_{I F}^{0}$ there, $k^{\prime}$ is a force constant assumed independent of $\left(q_{1} \cdots q_{N-1}\right)$. Then $\mid\left(\partial\left(U_{\beta}-U_{\alpha}\right) / \partial q_{N} \mid\right.$ equals $k^{\prime} \alpha$. Further, $Q_{N}$ $\int \exp \left[-k^{\prime} q_{N}^{2} / 2 k T\right] d q_{N},{ }^{22}$ where $q_{N}=0$ is the value of $q_{N}$ on the intersection hypersurface. Thus, $Q_{N}$ equals $\sqrt{2 \pi k T / k^{\prime}}$ and so $\left\langle S^{-1}\right\rangle / Q_{N}$ equals $1 / \sqrt{2 \pi k T k^{\prime} a^{2}}$. One can express this quantity in terms of $\lambda_{0}$, by noting that $\lambda_{0} / 4$ in Eq. (23) is the energy barrier when $\Delta G_{I F}^{0}=0$. For the simple quadratic model for $U_{\alpha}$ and $U_{\beta}$ one finds that $\lambda_{0} / 4$ equals $k^{\prime} a^{2} / 8$. Thus, we have

$$
\frac{\left\langle S^{-1}\right\rangle}{Q_{N}}=\frac{1}{\sqrt{4 \pi \lambda_{0} k T}} .
$$

In the actual many-dimensional case the values of $U_{\alpha}$ and $U_{\beta}$ on any $N-1$ dimensional hypersurface $\left(\right.$ fixed $\left.q_{N}\right)$ when suitably averaged at that $q_{N}$, depend approximately quadratically on a suitably chosen $q_{N}$, when one makes a "dielectrically unsaturated" approximation. ${ }^{3}$ One can show that Eq. (24) is approximately valid for this many-dimensional system also. 26

Equations (21)-(24) yield

$$
\begin{aligned}
k(T)= & \frac{2 \pi V^{2}}{\hbar} \sum_{I, F} \frac{|\langle F \mid I\rangle|^{2} e^{-E_{I} / k T}}{Q_{I}\left(4 \pi \lambda_{0} k T\right)^{1 / 2}} \\
& \times \exp \left[-\frac{\lambda_{0}}{4 k T}\left(1+\frac{\Delta G_{I F}^{0}}{\lambda_{0}}\right)^{2}\right]
\end{aligned}
$$

an equation used in several recent articles. ${ }^{4-6}$

We have noted earlier that in the rate expression for nonadiabatic transfers, e.g., Refs. 7 and 10 , an exponential containing $\Delta E^{0}$ instead of the $\Delta G^{0}$ in Eq. (25) has been sometimes written. In derivations of the latter result it was as- sumed that all the classical-like coordinates were equivalent to harmonic oscillators, and, as already noted, for such systems, $\Delta S^{0}$ vanishes and so $\Delta E^{0}$ and $\Delta G^{0}$ become equal for the present reaction. ${ }^{12}$

Equation (25) is the rate constant at a fixed separation distance $R$. If the reaction involves, instead, contributions from a distribution of $R$ 's one can proceed as in Ref. 3. One would obtain Eq. (25), but now instead of $\Delta G_{I F}^{0}$ one finds $\Delta G_{I F}^{0}$, equal to $\Delta G_{I F}^{0}+w^{p}-w^{r}$, where $w^{r}$ and $w^{p}$ denote the work required to bring reactants together and the products together, respectively, to some average distance $R$ in the transition state. One also finds that Eq. (25) is multiplied by a factor $4 \pi R^{2} \delta R \exp \left(-w^{r} / k T\right)$, where $\delta R$ is the range of separation distance $R$ contributing significantly to the reaction rate. An elaboration, in which a somewhat slow diffusion may be involved, is described in Ref. 5 .

In obtaining Eqs. (14)-(21), all stationary phase points (10) were taken to be real, since the aim in the paper was to treat the $q_{k}$ 's classically. One quantum correction is that of nuclear tunneling along the $q$ 's. This tunneling occurs when one or more of the $p_{k}$ 's in Eq. (10) is complex valued. In that case a factor $\exp \left(-2\left|\operatorname{Im} W_{f i}\right|\right)$ appears in the integrand of Eq. (14) (and in later equations), $\operatorname{Im} W_{f i}$ being the imaginary part of $W_{f i}{ }^{27}$ at this complex-valued stationary phase point. Additionally, some subleties appear in the sum $\Sigma$ in Eq. (16) and hence in the subsequent equations. ${ }^{28}$

In concluding this section we comment on the relation between Eq. (18) and an interesting paper of Schmidt. ${ }^{29} \mathrm{He}$ employed Yamamoto's ${ }^{30}$ expression for the rate constant in terms of the reactive flux correlation function and introduced a classical approximation by a different procedure. To see the connection between that paper and Eq. (18) we begin the present Eq. (4), the $\langle F \mid I\rangle$ being absent in Ref. 29 since the intent there was to convert all coordinates to classical. After replacing the delta function in (4) by its Fourier integral represention and noting that $\epsilon_{i}|i\rangle$ and $\epsilon_{f}|f\rangle$ equal $H_{i}|i\rangle$ and $H_{f}|f\rangle$, the $H$ 's being Hamiltonians, one finds

$$
k_{\alpha \beta}=\frac{V^{2}}{\hbar}|\langle F \mid I\rangle|^{2}\langle i \mid f\rangle \int_{-\infty}^{\infty}\left\langle f\left|e^{i H_{f} t} e^{-i H_{i} t}\right| i\right\rangle e^{i \Delta E_{F f}^{0} t} d t .
$$

To obtain $k_{I F}(T)$ as in Ref. 29, one sums over all final states $f$, uses the completeness relation to remove the $\Sigma_{f}|f\rangle\langle f|$, multiplies by a Boltzmann factor $\exp \left(-\beta \epsilon_{i}\right) / Q$, and sums over $i$. A classical-like approximation is then introduced ${ }^{29}$ by neglecting the commutator of $H_{f}$ and $H_{i}$ in Eq. (26). One obtains

$$
\begin{aligned}
k_{I F}(T)= & \frac{V^{2}}{\hbar Q}|\langle F \mid I\rangle|^{2} \\
& \times \sum_{i}\left\langle i\left|\int_{-\infty}^{\infty} e^{\left(H_{j}-H_{i}+\Delta E_{I F}^{0}\right) t} d t\right| i\right\rangle e^{-\beta \epsilon_{i}} .
\end{aligned}
$$

$H_{f}-H_{i}$ equals $U_{\beta}-U_{\alpha}$, and a second use of the integral representation for the delta function then yields an expression frequently used in the physics literature ${ }^{31}$

$$
\begin{aligned}
k_{I F}(T)= & \frac{2 \pi V^{2}}{\hbar Q}|\langle F \mid I\rangle|^{2} \\
& \times \sum_{i}\left\langle i\left|\delta\left(U_{\beta}-U_{\alpha}+\Delta E_{I F}^{0}\right)\right| i\right\rangle e^{-\beta \epsilon_{i}} .
\end{aligned}
$$

Because of the neglect of the commutator this type of expres- 
sion is sometimes termed "semiclassical"31 but it differs from the semiclassical approximation used in the present paper.

Schmidt then replaces the trace in Eq. (28), i.e., the sum over $i$, by an integral over phase space, and so obtains the desired classical expression. His procedure, beginning with Eq. (4), is clearly very direct. Its one disadvantage is that at an intermediate stage, namely Eq. (28), it can lead to a significant error, seen as follows: Equation (28) has been shown, ${ }^{32}$ when all coordinates are treated as harmonic oscillators, to lead to another ${ }^{11}$ expression for the reaction rate. That expression can be inaccurate (calculated ${ }^{32}$ to be a factor of $\sim 20$ different from the quantum value for the rate constant of thermoneutral reactions such as the $\mathrm{Fe}^{2+/ 3+}$ aquoion exchange at room temperature). The physical nature of the approximation has been identified, ${ }^{32,33}$ and one finds that Eq. (28) overestimates the quantum effect. ${ }^{34}$

\section{SUMMARY}

Microcanonical and canonical rate constants are derived for a nonadiabatic transition using generalized coordinates for the classical-like degrees of freedom. The results are used to derive Eq. (25), previously used in several studies in the nonadiabatic electron transfer literature. ${ }^{4-6}$ In Eq. (25) $\Delta G^{\circ}$ plays a major role even though it is $\Delta E^{0}$ which appears in the Golden Rule expression (1).

\section{ACKNOWLEDGMENT}

It is a pleasure to acknowledge support of this research by the National Science Foundation.

\section{APPENDIX A: RELATION BETWEEN COEFFICIENTS $a_{i j}$ and $g_{i j}$ AND APPLICATION TO EQ. (18)}

We consider the coefficients ${ }^{20} a_{i j}$ and $g_{i j}$ referred to in the text. An infintesimal element of length $d s$ is given in terms of curvilinear coordinates $\left(q^{1}, \ldots, q^{N}\right)$ by

$$
d s^{2}=\frac{1}{2} \sum_{i} \sum_{j} a_{i j} d q^{i} d q^{i}
$$

where

$$
a_{i j}=\sum_{k} \frac{\partial x^{k}}{\partial q^{i}} \frac{\partial x^{k}}{\partial q^{j}},
$$

and the $x^{k}$ 's are Cartesian coordinates. The usual covariantcontravariant notation. ${ }^{20}$ is used. (We drop this notation in all other sections of the paper.) The kinetic energy is given, on the other hand, by

$$
\mathrm{KE}=\frac{1}{2} \sum_{i j} g_{i j} \dot{q}^{i} \dot{q}^{j}=\frac{1}{2} \sum g^{i j} p_{i} p_{j},
$$

where

$$
g_{i j}=\sum_{k} m_{k} \frac{\partial x^{k}}{\partial q^{i}} \frac{\partial x^{k}}{\partial q^{j}},
$$

and $m_{k}$ is the mass associated with Cartesian coordinate $x^{k}$.

Equation (A1) leads to the following relation between the determinants:

$$
\left|a_{i j}\right|=\left|\sum_{k} \frac{\partial x^{k}}{\partial q^{i}} \frac{\partial x^{k}}{\partial q^{j}}\right|=\left|\frac{\partial x^{k}}{\partial q^{i}}\right|^{2}
$$

using the usual product rule. (In the text we have denoted determinants such as $\left|\partial x^{k} / \partial q^{i}\right|$ by $|\partial x / \partial q|$.) Equation (A4) leads to

$$
g_{i j}=\left|m_{k} \frac{\partial x^{k}}{\partial q^{j}}\right|\left|\frac{\partial x^{k}}{\partial q^{i}}\right|=\left|a_{i j}\right| \prod_{k} m_{k} .
$$

$\left|g_{i j}\right|$, which equals the reciprocal of $\left|g^{i j}\right|$, is denoted conventionally as $g$. Denoting $\left|a_{i j}\right|$ by $a$, Eqs. (A5) and (A6) yield

$$
1 /\left|g^{i j}\right|=g=a \prod_{k=1}^{N} m_{k},
$$

a result used below and in Appendix B.

We have considered the following momentum integral in treating Eq. (18):

$$
I=\int \cdots \int \exp \left(-\frac{1}{2} \sum_{i j} g^{i j} p_{i} p_{j} / k T\right) d p_{1} \cdots d p_{N} .
$$

Integration over the $p_{j}$ 's and use of Eq. (A7) yields $(2 \pi k T)^{N / 2} \sqrt{g}$, and hence $\sqrt{\mathrm{a}} \Pi_{k}\left(2 \pi m_{k} k T\right)^{1 / 2}$. Introducing this result into the integral in the numerator in Eq. (18) and into the $Q$ there then yields Eq. (19).

\section{APPENDIX B: RELATION OF EQ. (19) TO THE LANDAU- ZENER FORMULA}

It is useful to consider the connection between Eq. (19) and that obtained using the one-dimensional Landau-Zener formula for nonadiabatic transitions, since the differences between Eq. (19) and the adiabatic expression are then understood in a simple way. (See Ref. 6 for closely related derivation.)

For weak electronic interactions (small matrix element $V$ ) the Landau-Zener probability $P$ of transition from electronic state $\alpha$ to state $\beta$ is given by ${ }^{35}$

$$
P=4 \pi V^{2} / \hbar v_{x} S_{x}
$$

where $v_{x}$ is the (Cartesian) velocity $\dot{x}$ at the crossing point and $S_{x}$ is the difference of slopes of the two potential energy curves at that point, the slope being expressed in Cartesian coordinates $S_{x}=d\left(U_{\beta}-U_{\alpha}\right) / d x$. The product $v_{x} S_{x}$, i.e., $(d x / d t)\left[d\left(U_{\beta}-U_{\alpha}\right) / d x\right]$ canalsobewrittenas $d\left(U_{\beta}-U_{\alpha}\right) /$ $d t$, and hence as $\dot{q}_{N} \partial\left(U_{\beta}-U_{\alpha}\right) / \partial q_{N}$, since $U_{\beta}-U_{\alpha}$ depends on only $q_{N}$ by definition of this coordinate in the text. Systems initially on surface $\alpha$ cross the intersection of the two potential energy curves, and if they do not go to $\beta$, reach a classical turning point on $\alpha$ and recross that intersection surface. Equation (B1) includes this probability of a double crossing.

The reaction rate constant $k(T)$ is equal to $P_{\alpha}$, the probability of finding the system in a phase space volume element at the crossing point, per unit length along the reaction coordinate, namely by

$$
P_{\alpha}=e^{-\epsilon / k T} d q_{1} \cdots d q_{N-1} d p_{1} \cdots d p_{N} / Q h^{N},
$$

multiplied by the velocity $\dot{q}_{N}$ and by the $P$ given by Eq. (B1), then integrated over all coordinates $\left(q_{1} \cdots q_{N-1}\right)$ on the $N-1$ dimensional surface of intersection of $U_{\alpha}$ and $U_{\beta}+\Delta E_{I F}^{0}$, and integrated over all momenta, noting that the $\epsilon$ in Eq. (B2) equals $U_{\alpha}+\frac{1}{2} \Sigma_{i j} g^{i j} p_{i} p_{j}$. (Even $p_{N}$ is allowed to be positive and negative since the system crosses the intersection hypersurface twice, in the low transition probability limit, for each 
crossing in the forward direction.) Using Eq. (A7) of Appendix A one obtains

$$
k(T)=\frac{2 \pi V^{2}}{\hbar} \frac{\int \cdots \int e^{-U_{\alpha} / k T} S^{-1} \sqrt{a} d q_{1} \cdots d q_{N-1}}{\int \cdots \int e^{-U_{\alpha} / k T} \sqrt{a} d q_{1} \cdots d q_{N}} .
$$

If multiplied by the probability $|\langle F \mid I\rangle|^{2}$ Eq. (B3) yields Eq. (19) in the text for $k_{I F}(T)$. Of course, a main purpose of the present paper was to derive Eq. (25) systematically from the Golden Rule expression (1) and not to begin with the classical expression (B2).

${ }^{1}$ Recent reviews of the electron transfer literature include those of $N$. Sutin, Progr. Inorg. Chem. 30, 441 (1983); L. Eberson, Adv. Phys. Org. Chem. 18, 79 (1982); R. D. Cannon, Electron Transfer Reactions (Butterworths, Boston, 1980); D. DeVault, Quart. Rev. Biophys. 13, 387 (1980); J. U1strup, Charge Transfer Processes in Condensed Media, Lecture Notes in Chemistry, No.10 (Springer, New York, 1979). An early review is given in R. A. Marcus, Annu. Rev. Phys. Chem. 15, 155 (1964).

${ }^{2}$ R. A. Marcus, J. Chem. Phys. 24, 966 (1956)

${ }^{3}$ R. A. Marcus, J. Chem. Phys. 43, $679(1965)$.

${ }^{4} \mathrm{~S}$. Efrima and M. Bixon, Chem. Phys. 13, 447 (1976); their results were also utilized by R. P. Van Duyne and S. F. Fischer, Chem. Phys. 5, 183 (1974).

${ }^{5}$ R. A. Marcus and P. Siders, J. Phys. Chem. 86, 622 (1982).

${ }^{6}$ A. Warshel, J. Phys. Chem. 86, 2218 (1982).

'V. G. Levich and R. R. Dogonadze, Collect. Czech. Chem. Commun. 26, 193 (1961) (O. Boshko, Translator, Univ. of Ottawa).

${ }^{8}$ V. G. Levich, in Physical Chemistry, Volume 9B: Electrochemistry, edited by H. Eyring, D. Henderson, and W. Jost (Academic, New York, 1970), Chap. 12; R. R. Dogonadze, in Reactions of Molecules at Electrodes, edited by N. Hush (Wiley, New York, 1971), pp. 135ff.

${ }^{9}$ N. R. Kestner, J. Logan, and J. Jortner, J. Phys. Chem. 78, 2148 (1974). The authors were aware, of course, of the important role played by $\Delta G^{0}$ (e.g., in Refs. 2 and 3). I am indebted to Dr. Jortner for first calling the present $\Delta E^{0}$ problem to my attention.

${ }^{10} \mathrm{~J}$. Ulstrup, and J. Jortner, J. Chem. Phys. 63, 4358 (1975).

"J. J. Hopfield, Proc. Nat1. Acad. Sci. U.S.A. 71, 3640(1974); M. Redi and J. J. Hopfield, J. Chem. Phys. 72, 6651 (1980).

${ }^{12}$ (a) When $\Delta S^{0}=0, \Delta G^{0}=\Delta H^{0} . \Delta H^{0}$ is negligibly different from $\Delta E^{0}$ for reactions in solution. (b) The entropy of activation (and of reaction) has also been obtained for the particular case that all modes of motion are treated as harmonic oscillators, but of different frequencies for reactants and products, by R. R. Dogonadze, A. M. Kuznetsov, and M. A. Vorotyntsev, Phys. Status Solidi B 54, 125 (1972). Such an entropy change is expected to be relatively small in comparison with major changes resulting from changes in solvation and associated with restricting or freeing the orientations of solvent molecules.

${ }^{13} \mathrm{~J}$. C. Tully, in Dynamics of Molecular Collisions, Part B, edited by W. H. Miller (Plenum, New York, 1976), Chap. 5.

${ }^{14}$ (a) J. H. Van Vleck, Proc. Natl. Acad. Sci. U.S.A. 14, 178 (1928); (b) J. B. Keller, Ann. Phys. 4, 180 (1958); (c) V. A. Fock, Vest. Leningrad Univ. Ser. Fiz, Khim. 16, 67 (1959); Technical Translations 4, 53 (1960), No. 6017464; (d) V. P. Maslov, Zhur. Vychisl. Mat. Mat. Fiz. 1, 113, 638 (1961), [English translation: USSR Compt. Math. Math. Phys. 1, 123, 744 (1962)]; (e) R. Schiller, Phys. Rev. 125, 1109 (1962); (f) R. A. Marcus, J. Chem. Phys. 59, 5135 (1973); R. A. Marcus, Faraday Discuss Chem. Soc. 55, 34 (1973); (g) W. H. Miller, Adv. Chem. Phys. 25, 69, (1974); (h) 30, 77 (1976). To be sure, all of these articles tacitly or explicity assume that the phase of the semiclassical wave function $S_{i} / \hbar$ has a finite number of branches, i.e., it describes a "quasiperiodic" motion, or at least it assumes that the system can be approximated by such a description. Quasiperiodic and chaotic behavior are discussed in D. W. Noid, M. L. Koszykowski, and R. A. Marcus, Annu. Rev. Phys. Chem. 32, 267 (1981), and references cited therein.

${ }^{15}$ The usual expression has been modified to include in Eq. (5) the curvilinear factor $a^{-1 / 2}$ and the sum $\Sigma$. In our case some motions are oscillatory and some are not. Usually only a single term, or in the one-dimensional oscillatory case, two terms, are written in the literature. The fact that many branches and hence many terms in $\Sigma$ may exist is, of course, well known [Ref. 14(b)].

${ }^{16}$ If $i_{k}$ is a quantum number, the relevant classical action is actually $\left(i_{k}+\delta\right) h$, rather than $i_{k} h$, where $\delta=0$ for a rotation and $1 / 2$ for a vibration. However, the value of $\delta$ does not affect any of the equations used here, since they involve differentials, such as $d i_{k}$, or differences, such as $i_{k}-i_{k}^{\prime}$.

${ }^{17}$ Examples of "primitive" semiclassical calculations of Franck-Condon factors in the literature include L. D. Landau and E. M. Lifshitz, Quantum Mechanics, 3rd ed. (Pergamon, New York, 1977), Sec. 51; W. H. Miller, Ref. 14(h); M. S. Child, in Semiclassical Methods in Molecular Scattering and Spectroscopy, edited by M. S. Child (Reidel, Boston, 1979), p. $127 \mathrm{ff}$.

${ }^{18}$ The argument leading from the various determinants in Eq. (12) to the one in Eq. (13) parallels that in Ref. 14(e) and 14(h): Differentiation of the stationary phase condition $(10)$ with respect to $f_{l}$ yields

$$
\sum_{j} \frac{\partial^{2}}{\partial q_{k} \partial q_{j}}\left(S_{f}-S_{i}\right) \frac{\partial q_{j}}{\partial f_{i}}+\frac{\partial^{2} S_{j}}{\partial q_{k} \partial f_{i}}=0 .
$$

$\left[q_{j}\right.$ depends on the $f$ 's and the $i$ 's, because of the stationary phase condition (10).] Hence, $\left|\partial^{2}\left(S_{f}-S_{i}\right) / \partial g^{2}\right||\partial q / \partial f|$ equals $\left|\partial^{2} S_{f} / \partial q \partial f\right|$, using the product rule for determinants. The ratio of determinants in Eq. (12) then becomes $\left|\partial^{2} S_{i} / \partial q \partial i\right||\partial q / \partial f|$. Since $\partial S_{i} / \partial q_{k}$ equals $p_{k}^{i}$, Eq. (13) follows. We note, parenthetically, that the determinants in Eq. (13) can be shown to equal $\left|\partial^{2} W_{f} / \partial i \partial f\right|$ : Using Eq. (10) $\partial W_{f} / \partial i_{k}$ equals $-\partial S_{i} / \partial i_{k}$, and hence $\partial^{2} W_{f i} / \partial f_{l} \partial i_{k}$ equals $-\Sigma_{k}\left(\partial^{2} S_{i} / \partial q_{j} \partial i_{k}\right)\left(\partial q_{j} / \partial f_{l}\right)$. Thereby, $\left|\partial^{2} W_{f} / \partial f \partial i\right|$ equals $\left|\partial^{2} S_{i} / \partial q \partial i\right||\partial q / \partial f|$, i.e., $|\partial p / \partial i||\partial q / \partial f|$.

${ }^{19}$ In the integration over $d\left(U_{\beta}-U_{\alpha}\right)$ the net result is positive, regardless of the sign of the slope, and hence the use of the absolute value sign in $\mathbf{E q}$. (15).

${ }^{20}$ R. A. Marcus, J. Chem. Phys. 41, 2624 (1964).

${ }^{21}$ R. A. Marcus, Discuss Faraday Soc. 29, 21 (1960).

${ }^{22} \mathrm{As}$ in Refs. 3 and 21 we choose the quantity now labeled as $U_{N}$ in Eq. (22) to be $m\left(U_{\alpha}-U_{\beta}-\Delta E_{i F}^{o}\right)$, [cf. Eq. (13) of Ref. 3, where the potentials were instead defined relative to the same zero], $m$ is a Lagrangian multiplier, which now depends on $I$ and on $F, m$ is chosen using a procedure analogous to that in the above references, but for the given $I$ and $F$, i.e., for the given $\Delta E_{I F}^{0}$ and hence for the given $\Delta G_{I F}^{0}$. The $Q_{N}$ in Eq. (21) is defined in the following way: The surface integral prior to the introduction of $U_{N}$ in Eqs. (21) and (22) was $\int \cdots \int \exp \left(-U_{\alpha} / k T\right) \sqrt{a} d q_{1} \cdots d q_{N-1}$ and is written asexp $\left[-I\left(q_{N}\right) / k T\right]$, defining thereby afree energy $I\left(q_{N}\right)$. Onemultiplies by $d q_{N}$, integrates over $q_{N}$, and expands $I\left(q_{N}\right)$ as a quadratic function of $q_{N}$ about its value $\left(q_{N}=0\right)$ on the intersection surface (Ref. 3). $Q_{N}$ is defined (Ref. 3) as the integral over this exponential and equals $\left[2 \pi I^{\prime \prime}(0) k T\right]^{1 / 2}, I^{\prime \prime}$ being the value of $d^{2} I / d q_{N}^{2}$ at the intersection hypersurface.

${ }^{23}$ One can see this comparing Eq. (19) [and Eq.(13)] of Ref. 3 with Eqs. (31) and $(81)$ there. The notation is somewhat different from the present notation.

${ }^{24}$ In Ref. $3 \lambda_{0}$ occurs in Eq. (68) and, by comparison with Eq. (89) there, is the coefficient of $m^{2}$ in Eq. (89).

${ }^{25}$ This contribution to $\lambda_{0}$ is labeled $\lambda_{i}$ in Ref. 3 and given by Eq. (84) there. ${ }^{26}$ We use the e.e.d. to calculate the averages of $U_{\alpha}$ and $U_{\beta}$ and describe these averages using the parameter $m$. This $m$ entered earlier (Ref. 22) into $U_{N}$ and like $q_{N}$, has played a major role (Ref. 21) in defining the position of the intersection hypersurface: $m$ is varied by varying $\Delta G_{I F}^{0}$ (Ref. 3 and 21). We write $d / d q_{N}$ as $(d / d m)\left(d m / d q_{N}\right)$. The difference, $U_{\beta}-U_{\alpha}$, averaged over an e.e.d. at a given $q_{N}$ (and hence at a given $m$ ) can be shown from Eq. 168 of Ref. 3 and from an analogous equation for the free energy of formation of the e.e.d., at this same $q_{N}$, on the $\beta$ surface from the products to be $(m+1)^{2} \lambda_{0}-m^{2} \lambda^{0}$, plus a term independent of $m$. That is, $\left\langle U_{\beta}-U_{\alpha}\right\rangle$ at this $q_{N}$ is $(2 m+1)^{2} \lambda_{0}-m^{2} \lambda^{0}$ plus an $m$-independent term. Hence, $\left\langle d\left(U_{B}-U_{\alpha}\right) / d q_{N}\right\rangle$ equals $2 \lambda_{0} d m / d q_{N}$. Further, the $q_{N}$-partition function $Q_{N}$ is approximately equal to that for the reactants, namely $\int \exp \left(-\Delta F_{0}^{*} / k T\right) d q_{N}$, where [Eq. (68)] $\Delta F_{0}^{*}$ is $m^{2} \lambda_{0}$. Integration of the latter over $q_{N}$ yields $\sqrt{\pi k T / \lambda_{0}} d q_{N} / d m$. Thus, the approximate value of $\langle S\rangle^{-1} / Q_{N}$ is $1 / \sqrt{4 \pi \lambda_{0} k T}$. While $\langle S\rangle^{-1}$ is not exactly equal to $\left\langle S^{-1}\right\rangle$ except when $S$ is a constant, as it was in the simple harmontic oscillator model, we see that approximately $\left\langle S^{-1}\right\rangle / Q_{N}$ equals $1 / \sqrt{4 \pi \lambda_{0} k T}$, as in Eq. (24).

${ }^{27}$ For example, in one dimension the exponential factor $\exp \left(-2\left|\operatorname{Im} W_{f}\right|\right.$ h) becomes $\exp (-2 J / \hbar)$ where, when the slopes $\partial U_{\alpha} / \partial q_{N}$ and $\partial U_{\beta} / \partial q_{N}$ are of opposite sign, $J$ is the sum of the absolute values of the phase integrals $\int p_{N} d q_{N}$ from the classical turning point on curve $\alpha$ to the crossing 
point and from the crossing point to the classical turning point on curve $\beta$. When the slopes are of the same sign, $J$ is the difference in absolute values [e.g., Eq. (51.6) of Ref. 17].

${ }^{28}$ The preexponential factor in the one-dimensional case (or in the case where the $q_{N}$ coordinate is separable from the others) is a factor of 2 smaller in this case than the factor in Eq. (B1) (also $v_{x}$ is replaced by $\left|v_{x}\right|$ ). This result is seen, for example, by comparing Eq. (B1) with the relevant probability factor, the second factor in brackets, in Eq. (14) of T. Holstein, Philos. Mag. B 37, 49 (1978). The origin of this difference is also evident in the semiclassical wave function in the classically allowed region, there being two exponential terms with imaginary exponents when a turning point occurs, while in the classically forbidden region there is only one term, a decaying exponential [cf. Eq. (51.2) of Ref. 17].

${ }^{29}$ P. P. Schmidt, J. Chem. Phys. 58, 4384 (1973), Eq. (16).
${ }^{30}$ T. Yamamoto, J. Chem. Phys. 33, 281 (1960).

${ }^{31}$ M. Lax, J. Chem. Phys. 20,1752 (1952); D. Curie, Luminesence in Crystals (Wiley, New York, 1963), p. 47ff. The following authors obtain the same expression by an expansion in semi-invariants: $R$. Kubo and $Y$. Toyozawa, Prog. Theor. Phys. 13, 160 (1955); K. Maeda, Phys. Chem. Solids 9, 335 (1959); T. F. Soules and C. B. Duke, Phys. Rev. B 3, 262 (1971).

${ }^{32}$ P. Siders and R. A. Marcus, J. Am. Chem. Soc. 103, 741 (1981).

${ }^{33}$ R. A. Marcus, in Oxidases and Related Redox Systems, edited by T. E. King, M. Morrison, and H. S. Mason (Pergamon, New York, 1982), p. 3.

${ }^{34}$ For related comments see J. Jortner, J. Chem. Phys. 64, 4860 (1976).

${ }^{35}$ L. Landau, Phys. Z. Sov. 1, 88 (1932); 2, 46 (1932); C. Zener, Proc. R. Soc. London Ser. A 137, 696 (1932). 